\title{
A microscopic complex potential description of elastic, inelastic cross section in the Coulomb nuclear interference region in the ${ }^{28} \mathrm{Si}$ on ${ }^{28} \mathrm{Si}$ system
}

\author{
Samit Mandal ${ }^{\mathrm{a}, \mathrm{b}, 1}$, T. Madhusoodhanan ${ }^{\mathrm{c}}$, Subinit Roy ${ }^{\mathrm{d}}$, S. Ray ${ }^{\mathrm{b}}$,
} H. Majumdar ${ }^{\text {d }}$, S. Datta ${ }^{\text {e }}$, V. Ram Devraj ${ }^{\text {f }}$, V.K. Mittal ${ }^{\text {g }}$, D. Kabiraj ${ }^{a}$, S. Ghosh ${ }^{\text {a }}$, A. Tripathi ${ }^{\text {a }}$, A. Mandal ${ }^{\text {a }}$, D.K. Avasthi ${ }^{\text {a }}$, S.K. Datta ${ }^{\text {a }}$

a Nuclear Science Centre, Post Box 10502, New Delhi 110067, India

${ }^{b}$ Dept. of Phys., University of Kalyani, Kalyani 741235, India

${ }^{c}$ Dept. of Phys., Bangalore University, Bangalore 560056, India

d Saha Inst. of Nucl. Phys., I/AF Bidhannagar, Calcutta 700064, India

${ }^{\mathfrak{e}}$ Dept. of Phys., University of Calcutta, Calcutta 700009, India

${ }^{\mathrm{f}}$ Dept. of Phys., Osmania University, Hyderbad 500007, India

g Dept. of Phys., Panjabi University, Patiala 147002, India

Received 1 May 1997; revised 15 January 1998; accepted 17 February 1998

\begin{abstract}
Elastic and inelastic angular distribution and excitation functions were measured for the ${ }^{28} \mathrm{Si}+$ ${ }^{28} \mathrm{Si}$ system in the vicinity of the Coulomb barrier. While the elastic data could be described very well by using fully microscopic complex potential, the inelastic cross sections were found to be more sensitive to small variations in the potential. In particular the Coulomb nuclear interference dip observed in the inelastic excitation functions could not be fitted satisfactorily with calculation. Inclusion of an energy dependent term of Gaussian shape to the associated matrix element with the reorientation coupling in the phenomenological calculations leads to a better fit the inelastic excitation functions. (c) 1998 Elsevier Science B.V.
\end{abstract}

PACS: $24.50 .+\mathrm{g} ; 25.70 . \mathrm{Bc} ; 27.60 .+\mathrm{j}$

Keywords: Nuclear reactions ${ }^{28} \mathrm{Si}\left({ }^{28} \mathrm{Si}^{28} \mathrm{Si}\right),\left({ }^{28} \mathrm{Si}^{28}{ }^{28} \mathrm{Si}^{\prime}\right), E=63,72 \mathrm{MeV}$; Measured $\sigma(\theta) ; \theta_{\text {c.m. }}=90^{\circ}$; $\sigma(E) ; 58-84 \mathrm{MeV}$ coupled-channels analysis with phenomenologically and microscopically obtained potentials

\footnotetext{
${ }^{1}$ Corresponding author. tel. +91 116893955 , fax +91 116893666 , e-mail samit@nsc.ernet.in.
} 


\section{Introduction}

Heavy-ion elastic scattering at low incident energies is generally well described by complex optical model potentials [1]. In a pure phenomenological optical model with parametrised Woods-Saxon form, one uses adjustable parameters to fit the observed cross section. However, because the elastic cross section is sensitive to the asymptotic phase shift only, there are ambiguities in the extraction of these parameters. Consequently, the appropriate potentials to be used with a reaction model for describing inelastic scattering or transfer reaction have to be arrived at from other considerations. In a microscopic model on the other hand, potentials are calculated from fundamental considerations and, therefore, such ambiguities do not arise. The folding model is one such widely and successfully used microscopic model for describing heavy-ion elastic scattering [2]. In such a model, the real part of the optical potential is derived by folding the nuclear density of the colliding nuclei with an effective nucleon-nucleon interaction. The imaginary part of the potential is still extracted phenomenologically.

Several attempts have been made to calculate microscopically the imaginary part of the complex optical potential. Broglia et al. [3,4] derived the imaginary potential semiclassically based on the trajectory of the colliding nuclei. Brink et al. [5,6] estimated the imaginary potential for the transfer channels using the proximity method based on the nucleus-nucleus interaction. Several authors used the complex energy density formalism $[7,8]$ based on the complex, two-body interaction to describe elastic scattering. Using the Brueckner-Hartree-Fock approximation and Reid's hard core nuclear-nuclear interaction, a complex optical potential was derived [9] and successfully used in explaining the elastic scattering data for several systems. Vinh Mau and her co-workers [10,11] derived the nucleus-nucleus potential from the basic Feshbach [12] formalism and analysed elastic scattering data for various systems (both spherical and deformed), e.g. ${ }^{16} \mathrm{O}+{ }^{208} \mathrm{~Pb}[13],{ }^{32} \mathrm{~S}+{ }^{40} \mathrm{Ca}[14],{ }^{35,37} \mathrm{Cl}+{ }^{24} \mathrm{Mg}$ [15]. A fairly good agreement was observed between the calculation and experiment.

In Vinh Mau's approach [10], the imaginary part of the complex optical potential was derived by considering all possible closed and open reaction channels through the closure approximation. But around the Coulomb barrier, only a few open channels may contribute significantly. It was shown by Pacheco et al. [14] that in the elastic scattering of ${ }^{32} \mathrm{~S}$ on ${ }^{24} \mathrm{Mg}$, inclusion of only the lowest energy states in projectile and target as open channels was sufficient to explain the data. However, elastic scattering is unlikely to be very sensitive to the absorptive potential near barrier. On the other hand, inelastic scattering or transfer reactions where the wave function plays an important role, will be more sensitive to the details of the potential. Also, such studies give more information on the structure and dynamics of the system.

It is well known that the Coulomb nuclear interference phenomenon is very sensitive to the heavy-ion potential [16] around the barrier. In the interference region, both Coulomb and nuclear wave functions are well matched and the interference minimum observed in the inelastic excitation function is directly related to the shape of the nucleus [17]. An earlier work, which studied the ${ }^{16} \mathrm{O}+{ }^{58} \mathrm{Ni}$ system, using a semi- 
classical model, obtained a reasonably good fit to the Coulomb nuclear interference data [16]. In the ${ }^{16} \mathrm{O}+{ }^{92} \mathrm{Zr}$ system, Takagui et al. [18] performed a coupled channel calculation with a phenomenologically fitted potential to explain the Coulomb nuclear interference region. They found that around the barrier the inelastic data could not be fitted satisfactorily within the framework of the collective model. The interference pattern could be reproduced by introducing an unusually strong reorientation coupling for the state (anomalous coupling). This reminds one of the anomalous behaviour of the optical potential near barrier (threshold anomaly).

It has been found that the diagonal term in the potential matrix, responsible for elastic scattering shows an energy dependence around the Coulomb barrier [19]. This is a consequence of coupling to different channels. The real and imaginary part of the potential are connected through a dispersion relation. It has been pointed out that the dispersion relation should be applicable to all the elements of the potential matrix and that the non-elastic transitions, which are in general described by the off-diagonal coupling terms in the same potential matrix, should also exhibit energy dependent behaviour near the barrier. Smithson et al. [20] showed that an energy dependent reorientation term for the $3^{-}$state of ${ }^{208} \mathrm{~Pb}$ (i.e. the static quadrupole moment of that state) is needed to explain their data around the barrier. A progressive increase in the value of the reorientation term with decreasing energy is observed. A structure dependent factor such as the reorientation term cannot possibly have any dependence on the projectile energy. The above observation actually indicates that the associated coupling potential (non-diagonal) has a different energy dependence from the diagonal part of the potential. This aspect also needs to be looked into in the context of the microscopic model.

In our present study, we made an attempt to understand the elastic and inelastic scattering of ${ }^{28} \mathrm{Si}$ on ${ }^{28} \mathrm{Si}$ using a microscopically derived potential (both real and imaginary) around the Coulomb barrier. We also studied the Coulomb nuclear interference as a more sensitive test of the microscopic model, where we have now included absorption in contrast to the earlier semi classical picture [16]. The advantage of using ${ }^{28} \mathrm{Si}$ is that it is a deformed system $\left(\beta_{2}=0.38\right)$ [21] and coupling to the first few excited states should be the major part of the absorption potential around the barrier. Reorientation effects may play a significant role also. An identical particle system has other features due to exchange effects which probably have not been studied in detail especially in a microscopic model. Also as far as our knowledge goes, mainly asymmetric systems have been chosen for such studies. Further, the detailed transition densities for s-d shell nuclei are available in literature. Wildenthal's [22] "universal sd" (USD) interaction has been found to be very successful in predicting energies and transition densities for sd shell nuclei. Thus microscopic, real transition potentials can be calculated reliably for ${ }^{28} \mathrm{Si}$. Most of the early works are based on a collective model description. Ferguson et al. [23] measured the elastic angular distribution around the Coulomb barrier. Emling et al. [24] measured the elastic and inelastic angular distribution of ${ }^{28} \mathrm{Si}$ on ${ }^{28} \mathrm{Si}$ at five different energies and explained them satisfactorily within the frame work of the diffraction and optical model with a phenomenological potential. 
The organization of the paper is arranged as follows. In Section 2 we describe the experimental method. In Section 3 is devoted to the analysis of elastic and inelastic angular distributions using phenomenological and microscopically derived potentials. The excitation functions for elastic and inelastic scattering using a microscopic potential are discussed in Section 4. Finally, we present our conclusions in Section 5.

\section{Experimental method}

The experiment was carried out using the 15UD Pelletron accelerator at Nuclear Science Centre, New Delhi. A ${ }^{28} \mathrm{Si}$ beam was produced in a SNICS ion source, accelerated (in the energy range 58-84 MeV), momentum analysed and allowed to impinge on a ${ }^{28} \mathrm{Si}$ target. Beam current was 6 to $10 \mathrm{pnA}$. The Si target was made by vacuum evaporation of $99.99 \%$ enriched ${ }^{28} \mathrm{Si}$ oxide with carbon backing. Thicknesses of the two $\mathrm{Si}$ oxide targets used were $30 \mu \mathrm{g} / \mathrm{cm}^{2}$ and $67 \mu \mathrm{g} / \mathrm{cm}^{2}$ as determined from the energy loss of $\alpha$-particles from a ${ }^{241} \mathrm{Am}$ source. The thickness of carbon backing was $10 \mu \mathrm{g} / \mathrm{cm}^{2}$.

Angular distributions and excitation functions of elastic and inelastic $\left(2^{+}, 1.78 \mathrm{MeV}\right)$ scattering were measured in a $150 \mathrm{~cm}$ diameter scattering chamber by detecting the scattered particles in silicon surface-barrier detectors. Five such detectors $(300 \mu \mathrm{m})$ were used and arranged in one of the two movable arms in the chamber. The solid angles subtended by the detectors were between 3.5-7.5 msr. Two monitor detectors $(300 \mu \mathrm{m})$ were placed symmetrically at $11^{\circ}$ on either side of the beam. The monitors subtended an angle of $0.2^{\circ}$ at the target. An entrance collimator of $2 \mathrm{~mm}$ diameter was used at the entrance port of the target chamber. The beam was properly steered to keep the elastic peak count in the two monitor detectors identical within 5\%.

The precise angle of the detectors was determined using the heavy ion scattering of identical particle system [25]. For such a system, the differential cross section is symmetric about $\theta_{\text {c.m. }}=90^{\circ}$, and produces an interference maximum for particles of integral spin (boson). Thus the angular scale is calibrated by locating this maximum. The accuracy in the angle determination is estimated to be less than $0.1^{\circ}$.

Angular distribution data were taken at 63 and $72 \mathrm{MeV}$, in the range of $15^{\circ}$ to $45^{\circ}$ with steps of $1^{\circ}$. Only elastic data were taken at the lower energy $(63 \mathrm{MeV})$. A few overlapping angles were used for different detectors to check the consistency of the data. The results of such measurements (properly normalised for solid angle) agreed within statistics. Excitation functions were measured for both elastic and inelastic $\left(2^{+}, 1.778\right)$ in the energy range 58 to $84 \mathrm{MeV}$ in steps of $1 \mathrm{MeV}(0.5 \mathrm{MeV}$ in the region of the Coulomb barrier) at $\theta_{\text {lab. }}=45^{\circ}$. A single surface-barrier detector with angular acceptance of $0.3^{\circ}$ was used. Standard electronics was used and data was recorded in a Micro-VAX computer with our data acquisition program ONLINE. Particle identification was not required since almost all competing reaction channels have high negative $Q$-value and did not interfere in the region of interest.

Fig. 1 shows a typical energy spectrum obtained. Overall energy resolution obtained was about $500 \mathrm{keV}$. The absolute cross-section values were obtained by normalising 


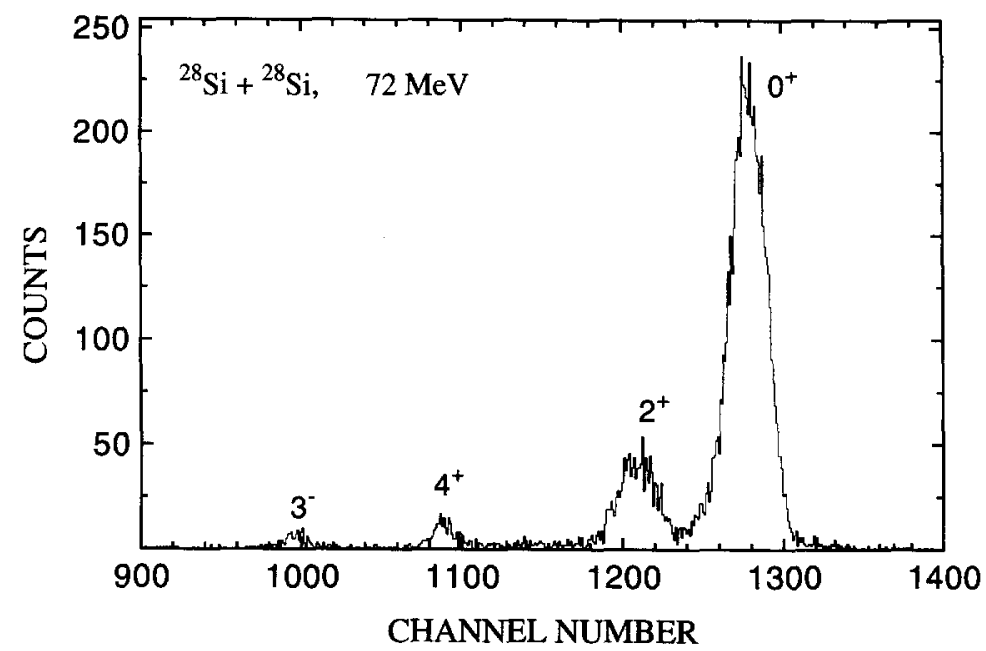

Fig. 1. Energy spectrum of particles from the elastic and inelastic scattering of ${ }^{28} \mathrm{Si}$ on ${ }^{28} \mathrm{Si}$ at $E_{\mathrm{lab}}=72 \mathrm{MeV}$ and $\theta_{\text {lat. }}=45^{\circ}$.

the yield to the elastic monitor counts, the cross section at the monitor angle being purely Coulombic and well described by the Mott scattering for spin zero particles. For the excitation function, data obtained during different runs were normalised from the overlapping energy points. The overall error was estimated to be less than $10 \%$ for elastic and less than $15 \%$ for inelastic cross sections.

\section{Analysis of angular distribution}

\subsection{Optical model analysis of elastic scattering}

A consistent optical model analysis was performed for the elastic scattering of 72 and $63 \mathrm{MeV}$ data. Optical model parameters were obtained by using the search option of the coupled channel code ECIS [26] in the uncoupled one-channel mode. We have considered the volume Woods-Saxon form for both the real and imaginary parts of the potential, i.e.

$$
U=-V f\left(r, R_{0}, a_{0}\right)-i W f\left(r, R_{w}, a_{w}\right),
$$

where

$$
f(r, R, a)=\left(1+\exp \left(r-\frac{R}{a}\right)\right)^{-1} .
$$

Here $V$ and $W$ are the strengths of the real and imaginary potentials respectively, with radius $R=r_{0}\left(A_{\mathrm{t}}^{1 / 3}+A_{\mathrm{p}}^{1 / 3}\right),\left(A_{\mathrm{t}}\right.$ and $A_{\mathrm{p}}$ being the masses of the target and projectile respectively) and diffuseness parameter $a$. The subscripts " 0 " and " $w$ " refer to real and imaginary parts respectively. 
Table 1

Optical potential parameters. The Coulomb radius parameter was $1.2 \mathrm{fm}$ in each case. The sets I and II refer to potentials obtained from the elastic channel, while set $\mathrm{CCI}$ and $\mathrm{CCII}$ are obtained from the simultaneous coupled channels fitting of the elastic and inelastic cross sections

\begin{tabular}{lcccccccc}
\hline Potential & $\begin{array}{c}E_{\mathrm{Lab}} \\
(\mathrm{MeV})\end{array}$ & $\begin{array}{c}V_{0} \\
(\mathrm{MeV})\end{array}$ & $\begin{array}{c}r_{0} \\
(\mathrm{fm})\end{array}$ & $\begin{array}{c}a_{0} \\
(\mathrm{fm})\end{array}$ & $\begin{array}{c}W \\
(\mathrm{MeV})\end{array}$ & $\begin{array}{c}r_{w} \\
(\mathrm{fm})\end{array}$ & $\begin{array}{c}a_{w^{\prime}} \\
(\mathrm{fm})\end{array}$ & $\chi^{2} / \mathrm{n}$ \\
\hline Set I & 63 & 95.41 & 1.21 & 0.49 & 12.63 & 1.18 & 0.4 & 4.5 \\
Set II & 72 & 77.59 & 1.21 & 0.49 & 22.47 & 1.18 & 0.4 & 4.9 \\
CCI & 72 & 80.55 & & & 17.08 & & & 4.2 \\
CCII & 72 & 6.47 & 1.406 & 0.612 & 4.64 & 1.344 & 0.359 & 2.5 \\
\hline
\end{tabular}

The best fit to the elastic scattering data was found by minimising the chi-squared $\left(x^{2}\right)$ value. It was observed that the fit was less sensitive to the variation of the optical potential parameters for $63 \mathrm{MeV}$ angular distribution data as expected below the barrier. Therefore, we carried out the search procedure on all the six parameters for the $72 \mathrm{MeV}$ data only. The geometry parameters were kept fixed after this. For the $63 \mathrm{MeV}$ data, we searched the real $(V)$ and imaginary strengths $(W)$ only, keeping the geometry parameters fixed. Final parameter sets obtained by this procedure (Table 1) are consistent with previous work [23,24].

It is known that several sets of parameters can fit the elastic data to about the same degree of accuracy, especially near the Coulomb barrier. In fact the potential in the interior regions (less than the strong absorption radius) is essentially undetermined. Attempts were made to find out some ranges of parameters that allow the chi-square per degree of freedom to vary within about $20 \%$ (from 4.9 per degree of freedom to 5.9 per degree of freedom on either side). One parameter was changed (in a grid search) and other parameters were also simultaneously varied to restore the fit within the above indicated values of chi-square. The real potential in set II could be varied $\sim \pm 18 \mathrm{MeV}$ from $77.59 \mathrm{MeV}$ in this manner. The imaginary potential too could be varied $\sim \pm 20 \mathrm{MeV}$ with a change in $\chi^{2}$ value of about the same magnitude. Similarly for $r_{0}, a_{0}, r_{w}$ and $a_{w}$, the ranges were $1.12-1.35 \mathrm{fm}, 0.43-0.54 \mathrm{fm}, 1.18-1.41 \mathrm{fm}$ (values of $r_{w}$ less than $1.18 \mathrm{fm}$ does not produce any change in $\chi^{2}$, when $W$ is simultaneously varied) and 0.05 to $0.90 \mathrm{fm}$. respectively. In all such cases the potential at strong absorption radius of $9.35 \mathrm{fm}$ remained practically identical (inset Figs. 3 and 5).

\subsection{Coupled channels calculation for inelastic scattering}

The inelastic angular distribution calculations were done using the same coupled channels code ECIS [26] with $0^{+}-2^{+}$coupling. Due to the symmetry of the system, the excitation to the $2^{+}$state for both target and projectile were included explicitly in the calculation. Elastic and inelastic $\left(2^{+} ; 1.778 \mathrm{MeV}\right)$ angular distributions were fitted simultaneously to extract the real and imaginary strengths of the potential parameters. The geometry parameters were kept the same as obtained from the elastic angular distribution search procedure (Table 1). As expected, due to explicit channel coupling, 
Table 2

Deformation parameters and quadrupole moment measurements taken from the literature [21,27,28] (set I). Set II represents the values deduced from the experimental data

\begin{tabular}{cccccc}
\hline & $J^{\pi}$ & $\begin{array}{c}E^{*} \\
(\mathrm{MeV})\end{array}$ & $\beta_{i}^{N}$ & $\begin{array}{c}M(\mathrm{E} l) \\
(\mathrm{efm})\end{array}$ & $\begin{array}{c}Q_{2} \\
\left(\mathrm{e} \mathrm{fm}^{2}\right)\end{array}$ \\
\hline \multirow{2}{*}{ Set I } & $2^{+}$ & 1.778 & 0.33 & 18.3 & 16.3 \\
& $3^{-}$ & 6.879 & 0.23 & 59.9 & $\ldots$ \\
\multirow{2}{*}{ Set II } & $4^{+}$ & 4.618 & 0.12 & 159.6 & $\ldots$ \\
& $2^{+}$ & 1.778 & 0.386 & 18.3 & 16.3 \\
\hline
\end{tabular}

the imaginary potential is lower for the coupled channels calculation. We assumed the real and imaginary deformation lengths to be equal i.e $\delta^{V}=\delta^{W}$. The Coulomb deformation $\left(\beta_{0 l}^{c}\right)$ was calculated from the reduced matrix elements for $0 \rightarrow l$ transition, (where $l$ is the order of the multipole). This is related to the reduced electromagnetic transition rate $(B(\mathrm{E} l) \uparrow)$ by

$$
M(\mathrm{E} l)= \pm\left[\left(2 J_{i}+1\right) B\left(\mathrm{E} l ; J_{i} \rightarrow J_{f}\right)\right]^{1 / 2},
$$

where $J_{i}$ and $J_{f}$ are the spins of the initial and final states. For the $0^{+}$to $2^{+}$transition,

$$
\beta_{20}=\frac{4 \pi[B(E 2)]^{1 / 2}}{3 Z R_{\mathrm{c}}{ }^{2}},
$$

with $Z=14$ for ${ }^{28} \mathrm{Si}$ and conventional target charge radius, $R_{\mathrm{c}}=1.2 A_{\mathrm{t}}{ }^{1 / 3}$. We have taken the value of the reduced matrix element $M(\mathrm{E} 2)$ equal to $18.3 \mathrm{e} \mathrm{fm}^{2}$ [21] and the nuclear deformation length as $1.22 \mathrm{fm}$ from the work of Zalmstra et al. [27]. Assuming a rotational model, the quadrupole moment for the excited $1.78 \mathrm{MeV}, 2^{+}$state was also taken into account through the reorientation coupling term $\beta_{22}$. $\beta_{22}$ was obtained from the static quadrupole moment value of the $2^{+}$state [28]. All the deformation parameters are shown in Table 2.

The elastic and inelastic cross sections from the coupled channels calculations along with the data are shown in Fig. 2a. The solid line represents the calculation with reorientation coupling and the dotted line is without reorientation. In the present work we have used the normally accepted oblate shape of ${ }^{28} \mathrm{Si}$ (i.e. a negative deformation was used). However, the interference pattern is not reproduced correctly with the potential set $\mathrm{CCI}$ and deformation set I. A variety of options were tried to fit the inelastic $2^{+}$ state data. By allowing both the potential strengths and the geometry parameters to vary a marginal improvement in the quality of the fit was observed. But the imaginary diffuseness and the real radius came out to be very low $\left(a_{w} \sim 0.09 \mathrm{fm}\right.$ and $r_{0} \sim$ $0.95 \mathrm{fm})$. In the next step we varied the nuclear deformation length with potential strengths and diffuseness parameters. We found a reasonable fit for the inelastic data with a large nuclear deformation length $\left(R_{0} \beta_{02}^{v}=1.65\right.$ and $\left.R_{w} \beta_{02}^{w}=1.58\right)$ and a shallow potential depth (see CCII in Table 1). The resulting fit is shown in Fig. 2b. The chisquared value for the inelastic angular distribution fit improved from 6.8 per degree of freedom to 4.3 , while the elastic fit remained practically identical for the parameter 

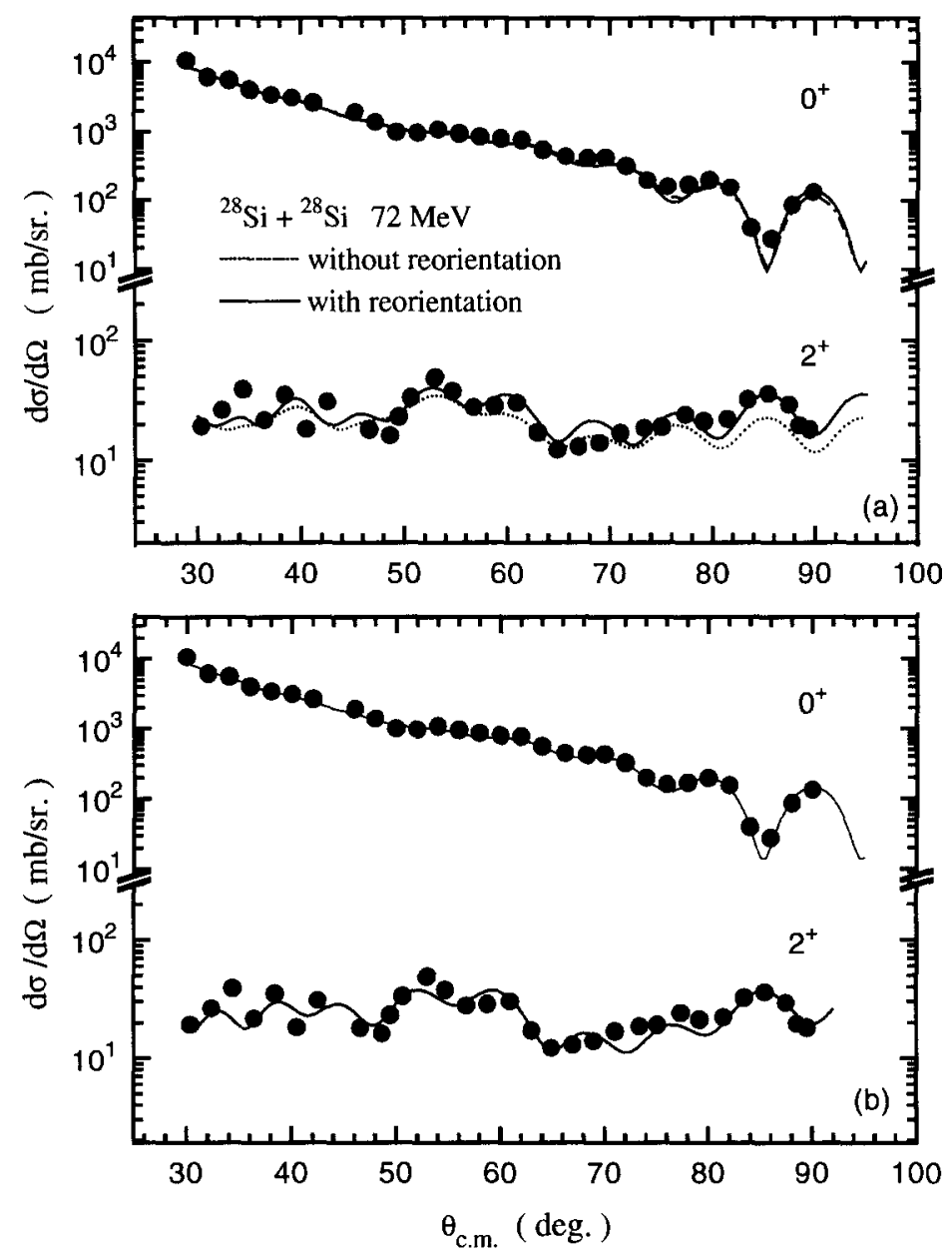

Fig. 2. Elastic and inelastic $\left(2^{+}, 1.778 \mathrm{MeV}\right.$ ) angular distributions for ${ }^{28} \mathrm{Si}+{ }^{28} \mathrm{Si}$. (a) Solid curves result from the coupled channels calculations with potential CCI (Table 1) including target and projectile excitations of first $2^{+}$state and quadrupole reorientation coupling (Table 2). Dotted line is without reorientation. The elastic curves for these cases are indistinguishable. (b) With the potential set CCII and the deformation parameter set II (see text).

sets $\mathrm{CCI}$ and CCII. We also varied the Coulomb deformation length $\left(R_{\mathrm{c}} \beta_{02}^{\mathrm{c}}\right)$ and the reorientation coupling strength $\left(\beta_{22}\right)$ one after another, but no further improvement could be obtained.

\subsection{Microscopic calculation}

While the fit using phenomenological optical potentials in a coupled channels description are good, our main interest was to obtain a description in a microscopic model with as few free parameters as possible. We proceeded in the following manner.

The real part of optical potential was calculated from a double folding between an 
effective (M3Y) two-body interaction and the density of the two interacting nuclei. The absorptive part of the potential was derived from the Feshbach formalism using the approach of Vinh Mau et al. [10,11] and Pacheco et al. [14]. The transition potential for the $2^{+}$excitation of the target and projectile nuclei was calculated by using the deformed folding model with a shell-model transition density [29].

\subsubsection{Real folded potential}

Our calculation is based on the generalised version of the folding model [30,31]. In this approach, the microscopic nucleus-nucleus potential is evaluated as an antisymmetrised Hartree-Fock type potential for the dinuclear system based on the first-order, many-body theory,

$$
V=V_{\mathrm{D}}+V_{\mathrm{EX}}=\sum_{i \in A_{1}, j \in A_{2}}\left[\left\langle i j\left|v_{\mathrm{D}}\right| i j\right\rangle+\left\langle i j\left|v_{\mathrm{EX}}\right| j i\right\rangle\right],
$$

where $|i\rangle$ and $|j\rangle$ are the single-particle, wave functions of two colliding nuclei $A_{1}$ and $A_{2}$ respectively. $V_{\mathrm{D}}$ and $V_{\mathrm{EX}}$ are the direct and exchange parts of the effective interaction. The widely used M3Y effective nucleon-nucleon (NN) interaction based on the $G$-matrix elements of the Reid soft-core interaction in an oscillator basis [32] with explicit density dependence to account for medium dependent effects (DDM3Y) was employed in our calculation. Due to the uncertainty involved in the derivation of an in-medium NN interaction from a realistic free $\mathrm{NN}$ interaction, a slight renormalisation of the folded potential, which gives the best fit to the data is commonly adopted [2]. The direct part of the interaction is,

$$
v_{\mathrm{D}}(r)=7999.0 \frac{\exp (-4 r)}{4 r}-2134.25 \frac{\exp (-2.5 r)}{2.5 r} .
$$

The exchange term can be a finite-range exchange,

$$
v_{\mathrm{EX}}(r)=4631.38 \frac{\exp (-4 r)}{4 r}-1787.13 \frac{\exp (-2.5 r)}{2.5 r}-7.8474 \frac{\exp (-0.7072 r)}{0.7072 r}
$$

The zero-range pseudopotential is also often used rather than the finite range exchange. It is of the form,

$$
v_{\mathrm{EX}}=-276\left(1-0.005 \frac{E_{\mathrm{lab} .}}{A_{2}}\right) \delta(\boldsymbol{r}) .
$$

In the case of the energy and density dependent M3Y interaction, DDM3Y, the potentials are

$$
\begin{aligned}
v_{\mathrm{D}(\mathrm{EX})}(\rho, E, r) & =F(\rho) g(E) v_{\mathrm{D}(\mathrm{EX})}(r), \\
g(E) & =1-0.002 E, \quad F(\rho)=C\left[1+\alpha e^{-\beta \rho}\right],
\end{aligned}
$$

where the density parameters are $C=0.2845, \alpha=3.6391$ and $\beta=2.9605 \mathrm{fm}^{3}$. The overall density in the above equation was taken to be the sum of the densities of the 
Table 3

Parameters of ground state density obtained from literature [33]

\begin{tabular}{cccc} 
Shape & $c(\mathrm{fm})$ & $z(\mathrm{fm})$ & $w$ \\
\hline $3 \mathrm{pG}$ & 1.954 & 2.076 & 0.286 \\
\hline
\end{tabular}

two colliding nuclei at the midpoint of the internucleon separation. This is the frozen density approximation usually adopted in the folding model calculation.

$$
\begin{aligned}
F(\rho) & =F\left(\rho_{1}\left(r_{1}+\frac{s}{2}\right)\right)+F\left(\rho_{2}\left(r_{2}-s 2\right)\right), \\
s & =r_{2}-r_{1}+R .
\end{aligned}
$$

The exchange potential $V_{\mathrm{Ex}}$ accounts for the knock-on exchange of nucleons between the projectile and the target. It is non-local in nature. By introducing the one-body density matrix $\rho_{1(2)}\left(r, r^{\prime}\right)$ of the two colliding nuclei and after certain transformations the final form of explicit energy dependent direct and self-consistent, local exchange potential can be written as

$$
\begin{aligned}
V_{\mathrm{D}}(E, \boldsymbol{R})= & \int \rho_{1}\left(\boldsymbol{r}_{1}\right) \rho_{2}\left(\boldsymbol{r}_{2}\right) v_{\mathrm{D}}(\rho, E, s) d^{3} r_{1} d^{3} r_{2}, \\
V_{\mathrm{EX}}(E, \boldsymbol{R})= & 4 \pi g(E) \int_{0}^{\infty} v_{\mathrm{EX}}(s) s^{2} d s j_{0}\left(\frac{k(\boldsymbol{R}) s}{\mu}\right) \\
& \times f_{1}(\boldsymbol{r}, s) f_{2}(\boldsymbol{r}-\boldsymbol{R}, s) F\left(\rho_{1}(\boldsymbol{r})+\rho_{2}(\boldsymbol{r}-\boldsymbol{R})\right) d^{3} r,
\end{aligned}
$$

where

$$
f_{1(2)}(r, s)=\rho_{1(2)}(r) \hat{j}_{1}\left(k_{F_{1(2)}}(r) s\right), \quad \text { and } \quad j_{0}(r)=\frac{\sin r}{r} .
$$

$k(\boldsymbol{R})$ is the relative momentum and $k_{\mathrm{F}}$ is the Fermi momentum. The exchange potential was calculated using a closed expression by a series expansion of Bessel function $j_{0}(k(\boldsymbol{R}) s / \mu)$.

Here we used the zero-range pseudopotential for calculating the $V_{\mathrm{EX}}$ term. It was observed that at low energies, the effect of finite-range exchange was very negligible for ${ }^{28} \mathrm{Si}$ on ${ }^{28} \mathrm{Si}$ system. The ground state density $\rho_{0}(\mathrm{r})$ was taken from the experimental charge density [33]. This charge density was parametrised as a three-parameter Gaussian $(3 \mathrm{pG})$ distribution (Table 3 ) which is of the form,

$$
\rho_{0}(r) \propto \frac{\left(1+w r^{2}\right)}{1+\exp \left(\frac{r^{2}-c^{2}}{z^{2}}\right)},
$$

where $c$ is the half-density radius, $z$ is the skin thickness and $w$ is in general influence of the tail of $\rho(r)$. Fig. 3 shows the calculated potential with a zero-range pseudopotential for $72 \mathrm{MeV}$ incident energy. In our calculations, we used corrections for the finite charge 

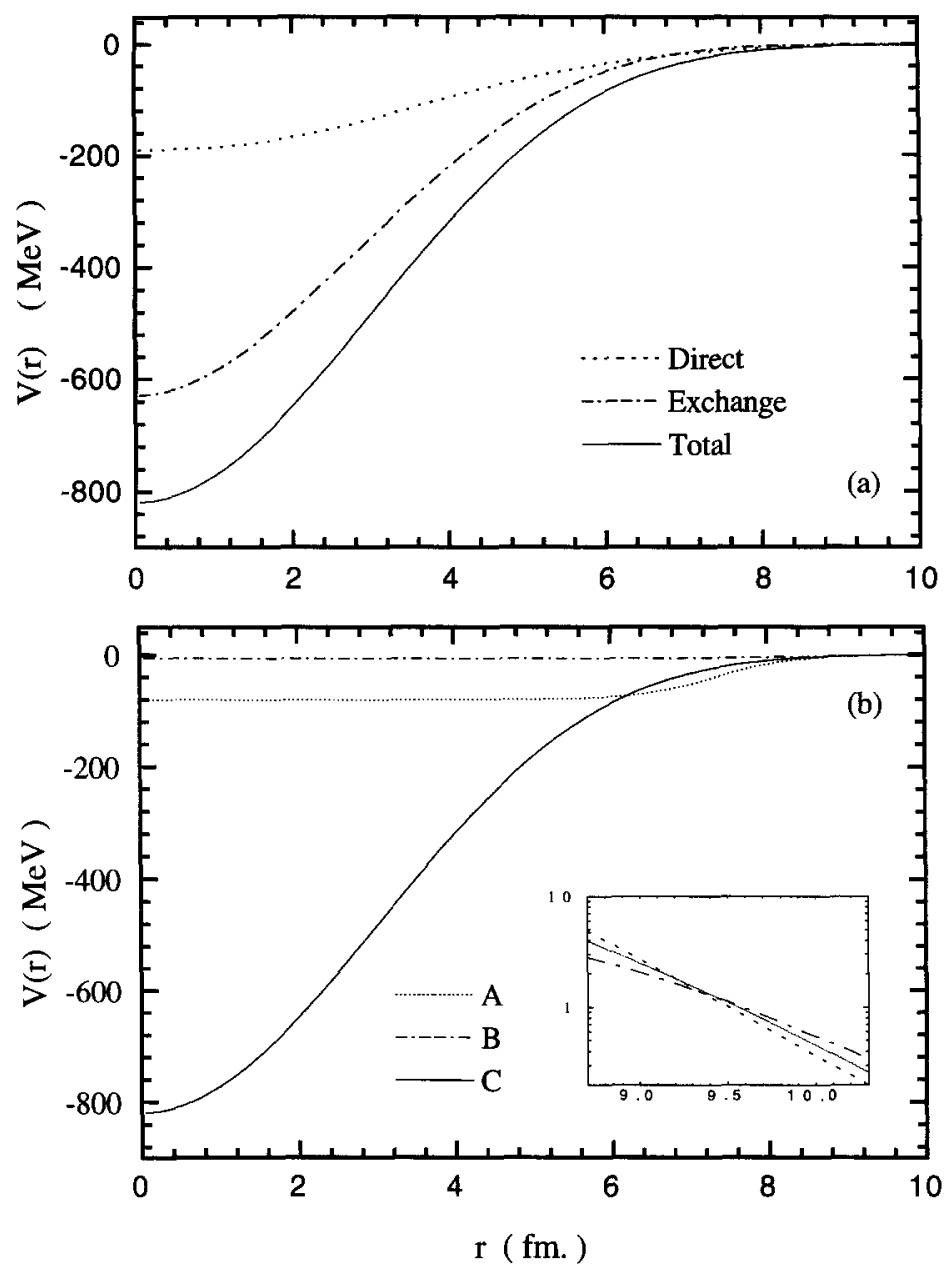

Fig. 3. Real folded potential for ${ }^{28} \mathrm{Si}$ on ${ }^{28} \mathrm{Si}$ system at $E_{\text {lab. }}=72 \mathrm{MeV}$, calculated with energy and density dependent M3Y interaction (DDM3Y). Exchange part (dash-dot curve) was calculated by taking DDM3Y interaction with zero range pseudopotential. Solid curve in the figure is the result of the contributions of direct (dotted curve) and exchange parts (top). The comparison between phenomenological and folded potentials are shown in (b) at the bottom. Where curves A and B are phenomenological potential from Table 1, set CCI and CCII respectively. The curve $\mathrm{C}$ is the double-folded potential.

distribution of the proton and took the shape of the neutron and proton distributions to be the same, the normalisation being in the $N / Z$ ratio.

\subsubsection{Real transition potential}

The microscopic transition density was calculated by expanding the nuclear density into multipoles $[2,1]$

$$
\rho(\boldsymbol{r})=\sum_{\lambda \mu} C_{\lambda}\left\langle I M \lambda \mu \mid L^{\prime} M^{\prime}\right\rangle \rho_{\lambda}(r) Y_{\lambda \mu}^{*}(\hat{\boldsymbol{r}})
$$


where $C_{0}=\sqrt{4 \pi}$ and $C_{\lambda}=1$ (if $\lambda \neq 0$ ). $\rho_{\lambda=2}(r)$ is the transition density from ground state $\left|0^{+}\right\rangle$to the excited state $\left|2^{+}\right\rangle$.

$$
\rho_{2}(r)=\left\langle 2^{+}\left\|\sum_{j} Y_{20}\left(r_{j}\right)\right\| 0^{+}\right\rangle .
$$

In our calculation of the transition potential for $2^{+}$excited state, the one-body density matrices (OBDM) were taken from Ref. [29] to generate the quadrupole transition density $\rho_{2}=\rho_{2}^{(p)}+\rho_{2}^{(n)}$ (for protons and neutrons). They derived the OBDM values for the ${ }^{28} \mathrm{Si}\left(0^{+}-2^{+}\right)$transition using the universal sd-shell effective interaction of Wildenthal [22] in the model space of sd shell. The transition density $\rho_{2}^{i}(i=\mathrm{p}, \mathrm{n})$ is written in terms of a valence part $A^{(i)}(r)$ and a core part $C^{(i)}(r)$. The former was explicitly calculated from the relation,

$$
A^{(i)}(r)=\sum_{j j^{\prime}} D_{j j^{\prime}}^{(i)} R(j, r) R\left(j^{\prime}, r\right)\left\langle j\left\|\sum_{j} Y_{\lambda \mu}\right\| j^{\prime}\right\rangle,
$$

where $D_{j j^{\prime}}$ are the one-body density matrices (OBDM) and $R(j, r), R\left(j^{\prime}, r\right)$ are radial single-particle wave functions. We have used a Wood-Saxon potential for the central part $\left(V_{0}=-51.41 \mathrm{MeV}, r_{0}=1.277 \mathrm{fm}, a_{0}=0.362 \mathrm{fm}\right)$, a standard spin-orbit potential $\left(V_{\mathrm{so}}=24.0 \mathrm{MeV}, r_{\mathrm{so}}=1.1 \mathrm{fm}, a_{\mathrm{so}}=0.65 \mathrm{fm}\right.$.) and a Coulomb potential for a proton in an orbit-independent method (for all levels in the sd-shell, the same potential was used) to generate the single-particle radial wave functions. These are extracted using the BOUND subroutine of the DWBA code DWUCKR4 [34]. The value of the reduced matrix elements of $Y_{\lambda, \mu}$ were taken from standard texts [35]. With these wave functions, the valence neutron and proton transition matrix elements were calculated and found to be $M_{\mathrm{n}}=10.1572 a \mathrm{e} \mathrm{fm}^{2}$ and $M_{\mathrm{p}}=10.499 \mathrm{e} \mathrm{fm}^{2}$. The effect of core polarisation was taken into account through the effective charges $e_{\mathrm{p}}=1.35$ and $e_{\mathrm{n}}=0.35$. The resulting total transition matrix elements for protons and neutrons are $M_{\mathrm{p}}=17.7286 \mathrm{efm}^{2}$ and $M_{\mathrm{n}}=17.3868 \mathrm{e} \mathrm{fm}^{2}$ respectively.

A double-folding procedure was used for the calculation with the energy-dependent DDM3Y type of interaction. In the case of single excitation of spin zero particles, the folded potential can be written (in momentum space) as

$$
V_{\lambda}(R)=\frac{1}{2 \pi^{2}} \int k^{2} d k j_{\lambda}(k R) \tilde{v}(k) \tilde{\rho_{\lambda}}(k) \tilde{\rho_{0}}(k) .
$$

The calculated transition potential is shown in Fig. 4. Around the strong absorption region $(9.35 \mathrm{fm})$, both the phenomenologically calculated transition potential and microscopically derived potential show similar behaviour.

\subsubsection{Absorptive potential}

The absorptive part of the potential was calculated following the approach of Vinh Mau and Pacheco et al. [10,14]. The generalised optical potential for elastic scattering at energy $E$ can be written as, 


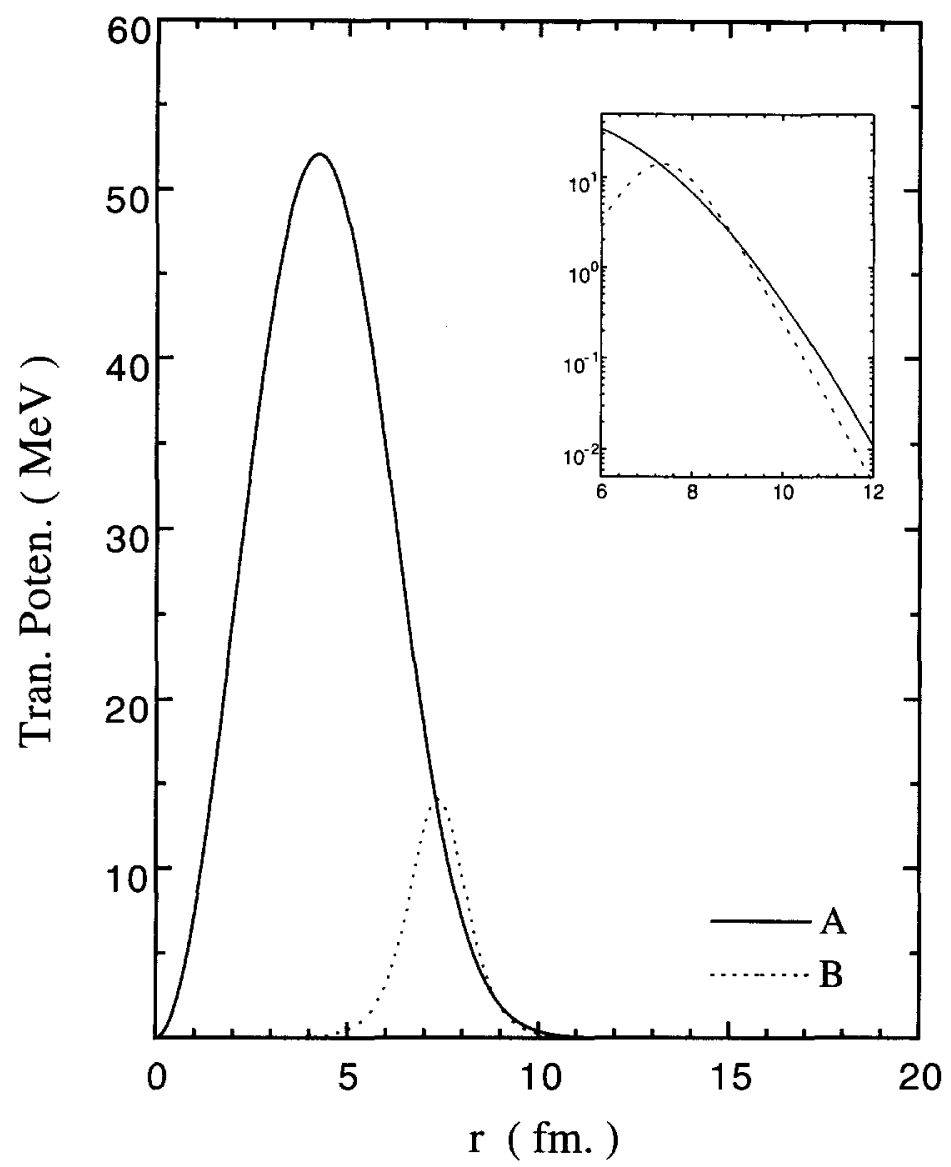

Fig. 4. Solid curve (curve A) represents the transition potential for ${ }^{28} \mathrm{Si}$ on ${ }^{28} \mathrm{Si}$ system at $E_{\text {lab. }}=45^{\circ}$ from folding model calculation using shell model transition density (with normalisation factor $1 / \sqrt{4 \pi}$. Dotted line (curve B) is from the deformed optical model potential $\left(=(1 / \sqrt{4 \pi}) \delta_{l}(\partial V / \partial r)\right)$. Logarithmic plot of the potential in the surface region to which the scattering is sensitive is shown in the inset.

$$
V_{\mathrm{opt}}\left(E ; r, r^{\prime}\right)=\left\langle\Phi_{0}|V| \Phi_{0}\right\rangle+\left\langle\Phi_{0}\left|V Q \frac{1}{E-H_{Q Q}+i \epsilon} Q V\right| \Phi_{0}\right\rangle=V_{0}+\Delta V
$$

where $\Phi_{0}$ is the ground state wave function, $Q$ is the projection operator which projects off the ground state, $H$ is the total Hamiltonian and $V$ is the interaction between the two colliding nuclei. $V_{0}$ is the double-folded potential and $\Delta V$ is the polarisation potential coming from the coupling of non-elastic channels to the elastic channel. The complex $\Delta V$ (as follows from the $i \epsilon$ term in the denominator) is also non-local and energy dependent in nature. In the weak coupling limit, one can write the polarisation potential as

$$
\Delta V\left(r, r^{\prime}\right)=\sum_{\alpha \neq 0} V_{\alpha}^{*}(r) G_{\alpha}\left(r, r^{\prime}\right) V_{\alpha}\left(r^{\prime}\right),
$$


where $\alpha$ denotes all possible channels. The $V_{\alpha}$ for a state $\alpha$ of angular momentum $\lambda$, $\mu$, can be written as

$$
V_{\alpha}(\boldsymbol{r})=\frac{1}{\sqrt{2 \lambda+1}} f_{\lambda}^{(\alpha)}(r) Y_{\lambda}^{\mu}(\boldsymbol{r})
$$

The $G_{\alpha}\left(r, r^{\prime}\right)$ is the Green function which can be calculated in the WKB approximation. After certain transformations, one can get the local equivalent polarisation potential explicitly for inelastic channels,

$$
\Delta V_{\text {in }}^{L}(R)=-\frac{\mu}{2 \pi \hbar^{2}} \sum_{\lambda \alpha \neq 0} \int_{0}^{2 R} s d s e^{i k_{r} s} j_{0}(k s) f_{\lambda}^{(\alpha)^{*}}\left(R+\frac{1}{2} s\right) f_{\lambda}^{(\alpha)}\left(R-\frac{1}{2} s\right),
$$

where $\boldsymbol{R}=\frac{1}{2}\left(\boldsymbol{r}+\boldsymbol{r}^{\prime}\right), \boldsymbol{s}=\boldsymbol{r}-\boldsymbol{r}^{\prime}$ and $K_{\alpha}$ is the WKB local momentum for the $\alpha$ channel. $K_{\alpha}^{2}=\frac{2 \mu}{\hbar^{2}}\left(E_{\mathrm{c} . \mathrm{m} .}-E_{\alpha}-V_{N}^{L}(R)-V_{\mathrm{c}}(R)\right)$ and $k^{2}=\frac{2 \mu}{\hbar^{2}}\left(E_{\mathrm{c} . \mathrm{m} .}-V_{N}^{L}(R)-V_{\mathrm{c}}(R)\right)$, $V_{N}^{L}(R)=V_{0}(R)+\Delta V_{L}(R)-i W_{L}(R)$, where $V_{N}^{L}(R)$ is the nucleus-nucleus potential and $V_{\mathrm{c}}$ the Coulomb potential.

For a low-lying collective state (as in the $2^{+}$state of ${ }^{28} \mathrm{Si}$ ) the nuclear form factor $f_{\lambda}^{(\alpha)}$ can be described by

$$
f_{\lambda}^{(\alpha)}(r)=\beta_{\lambda i}^{(\alpha)} R_{i} \frac{\partial U(r)}{\partial r}
$$

where $\beta_{\lambda i}^{(\alpha)}$ is the deformation parameter in the $\alpha$ channel for the nucleus $i$ of radius $R_{i}$ and $U(r)$ is the nucleus-nucleus potential. For mutual excitation (projectile and target simultaneously) the nuclear form factor can be written as

$$
f_{\lambda}^{(\alpha)}(r)=\frac{1}{\sqrt{4 \pi}} \beta_{\lambda i}^{(\alpha)} \beta_{\lambda j}^{(\alpha)} R_{i} R_{j} \frac{\partial^{2} U(r)}{\partial r^{2}}
$$

In our calculation the $V_{0}(R)$ was calculated from the double-folding model and $U(r)$ was taken from the Copenhagen potential [36].

Finally, one can obtain the total polarisation potential by the following equation through an iteration procedure.

$$
\begin{aligned}
\Delta V_{L}(R) & =\operatorname{Re} \Delta V_{\mathrm{in}}^{L}(R)+\operatorname{Re} \Delta V_{\mathrm{mut}}^{L}(R), \\
W_{L}(R) & =\operatorname{Im} \Delta V_{\mathrm{in}}^{L}(R)+\operatorname{Im} \Delta V_{\mathrm{mut}}^{L}(R)
\end{aligned}
$$

Using the above model, we calculate the imaginary potentials including only $2^{+}$ inelastic states for target, projectile excitation and the mutual contribution for $2^{+}$state (Fig. 5). The real part of the polarisation potential which includes all the open and closed channels contributions was then renormalised by a factor $\lambda$ (to incorporate the effects of those channels which are not taken into account explicitly in the calculation). So our total potential for the elastic scattering analysis was

$$
V=V_{0}(R)+\lambda \Delta V_{L}(R)+W_{L}(R)+V_{\mathrm{c}}
$$



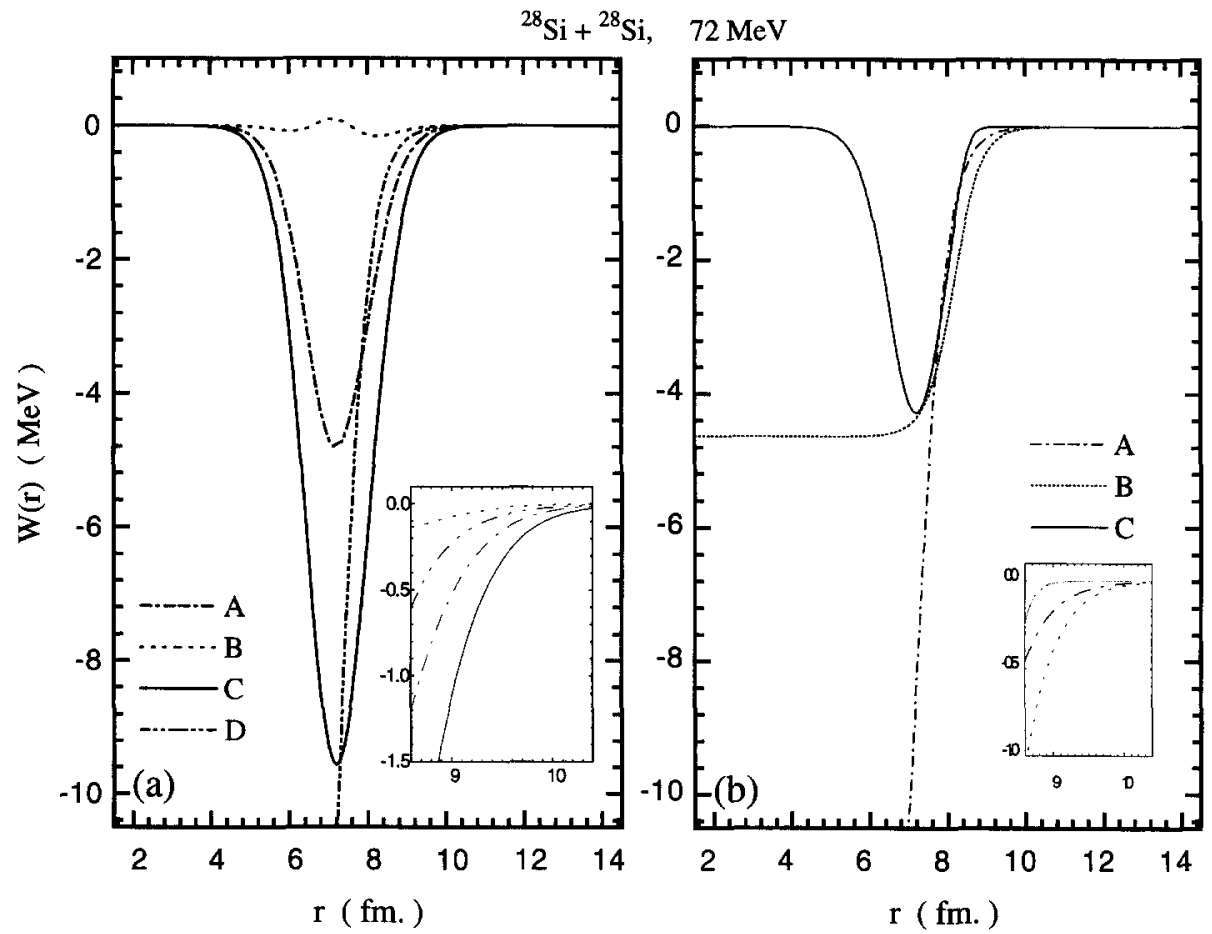

Fig. 5. (a) Imaginary potentials of ${ }^{28} \mathrm{Si}+{ }^{28} \mathrm{Si}$ at $E_{\text {lab. }}=72 \mathrm{MeV}$. Solid curve (curve C) in the figure is the total imaginary potential (see text) including the contribution of $2^{+}$state of target and projectile (dash-dot curve, A) and their mutual contribution (dotted curve, B). Curve D represents the phenomenological potential from Table 1, set II. (b) Imaginary potentials for coupled channels calculations $\left(0^{+}-2^{+}\right)$. Curve $\mathrm{C}$ is the microscopically calculated potential and curves $\mathrm{A}$ and $\mathrm{B}$ represent the phenomenological potential from Table 1, set CCII and CCI respectively.

Using this microscopic potential $(\lambda=1)$ we performed the elastic scattering calculation at $72 \mathrm{MeV}$ and $63 \mathrm{MeV}$. The nuclear deformation length $\delta=\beta_{20} R$ used was $1.22 \mathrm{fm}$ [27]. The calculated curve is shown in Fig. 6 by solid a line together with the elastic data. For both energies, the theoretical calculations agreed excellently with experiment. Introduction of other inelastic channels $\left(4^{+}, 3^{-}\right)$in the calculation produce a negligible improvement to the fit.

As the next step, we performed a coupled channel calculation using $0^{+}-2^{+}$coupling with both target and projectile excitation. A real double-folding potential, normalised real polarisation potential and a microscopically calculated imaginary potential, which included the $3^{-}$and $4^{+}$states only were used as the diagonal term of the potential. The real transition potential was derived from the shell model transition density as mentioned above and the imaginary part of the transition potential was calculated by taking the derivative of the imaginary potential used in the diagonal term of the potential multiplied by the deformation length $\left(\delta_{l}\right)$. Using this prescription the coupled channels calculations were performed with the coupled channels code FRESCO [37] which can include both inelastic and transfer channels. In our calculation, we included all the partial waves up 


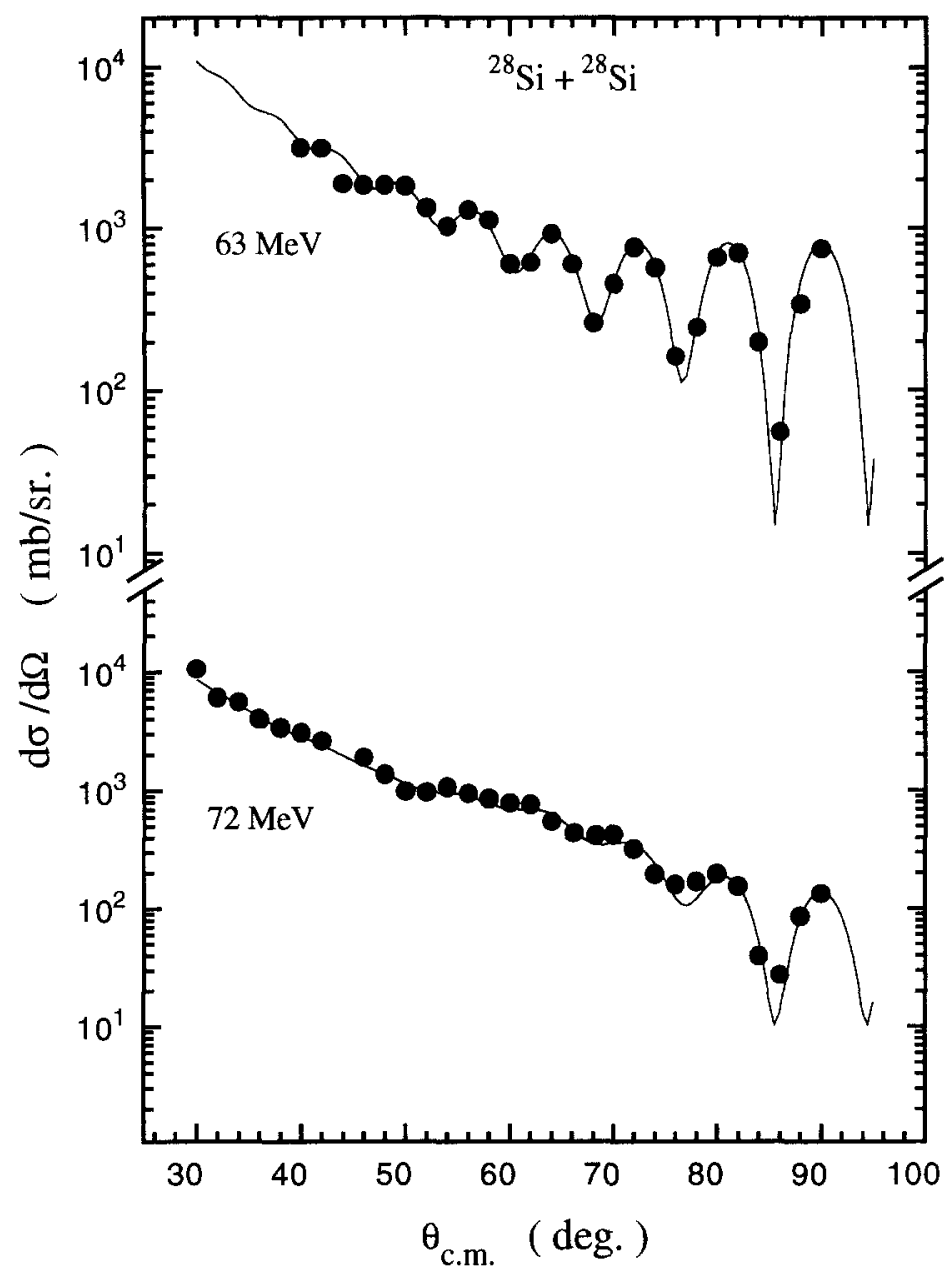

Fig. 6. Elastic angular distribution for ${ }^{28} \mathrm{Si}+{ }^{28} \mathrm{Si}$ system. Solid curve shows the theoretical predictions with microscopically calculated potential (see text).

to $l=250 \hbar$. The matching radius was set at $30 \mathrm{fm}$ for the convergence of the cross section and the integration step size was $0.05 \mathrm{fm}$.

The experimental data for the elastic channel are well reproduced by the theoretical calculation with and without quadrupole reorientation. In the case of inelastic scattering, the theoretically predicted cross section without reorientation coupling shows the interference pattern but does not describe the experimental data fully. Introduction of reorientation coupling terms in the calculation matches the oscillation pattern of the experiment at larger angles. It is also to be noted that the effect of the reorientation term in the elastic channel is negligible. By changing the sign of the quadrupole moment, i.e., the shape of the nucleus from oblate to prolate a distinctive effect on the inelastic channel was seen but nothing significant in the elastic channel. There is better agreement between calculation and experimental data with oblate deformation (Fig. 7). 


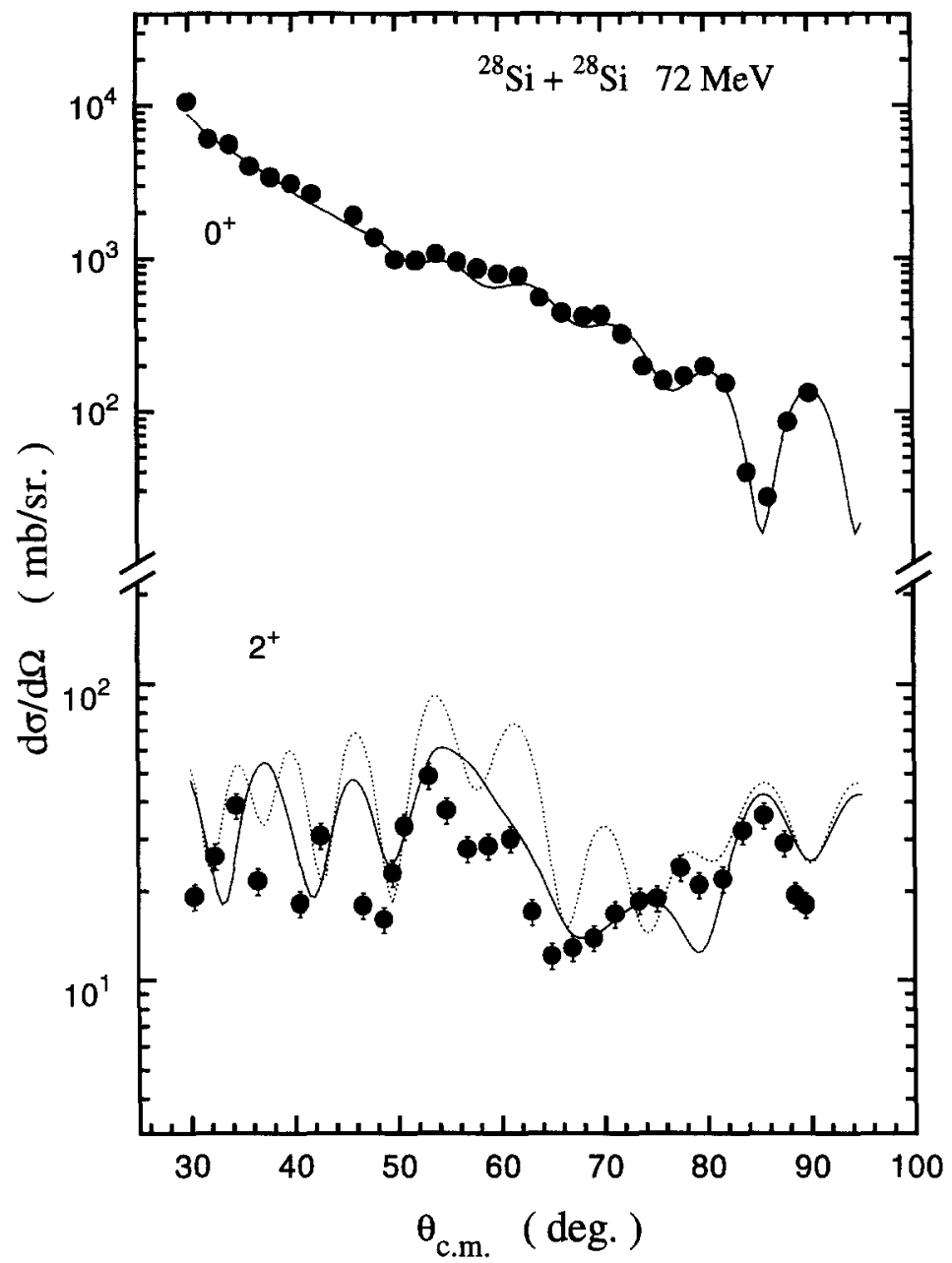

Fig. 7. Solid curve represents the coupled channels calculation with microscopically derived potential (see text) including $0^{+}-2^{+}$coupling of target and projectile excitations and quadrupole reorientation coupling (oblate shape). Dotted line with prolate shape.

\section{Excitation function}

Excitation functions for elastic and inelastic $\left(2^{+}\right.$state $)$scattering were also analysed using both the phenomenological optical model and the microscopic model.

\subsection{Elastic excitation function}

In the present study, we have seen that the microscopic potentials are reproducing the elastic scattering data well. With these potentials, we performed a detailed analysis of the elastic excitation function by taking into account the coupling of the first excited state $\left(2^{+}\right)$with the ground state using the coupled channels code FRESCO. For each energy point (at steps of $0.5 \mathrm{MeV}$ ) the microscopic imaginary potential was calculated 


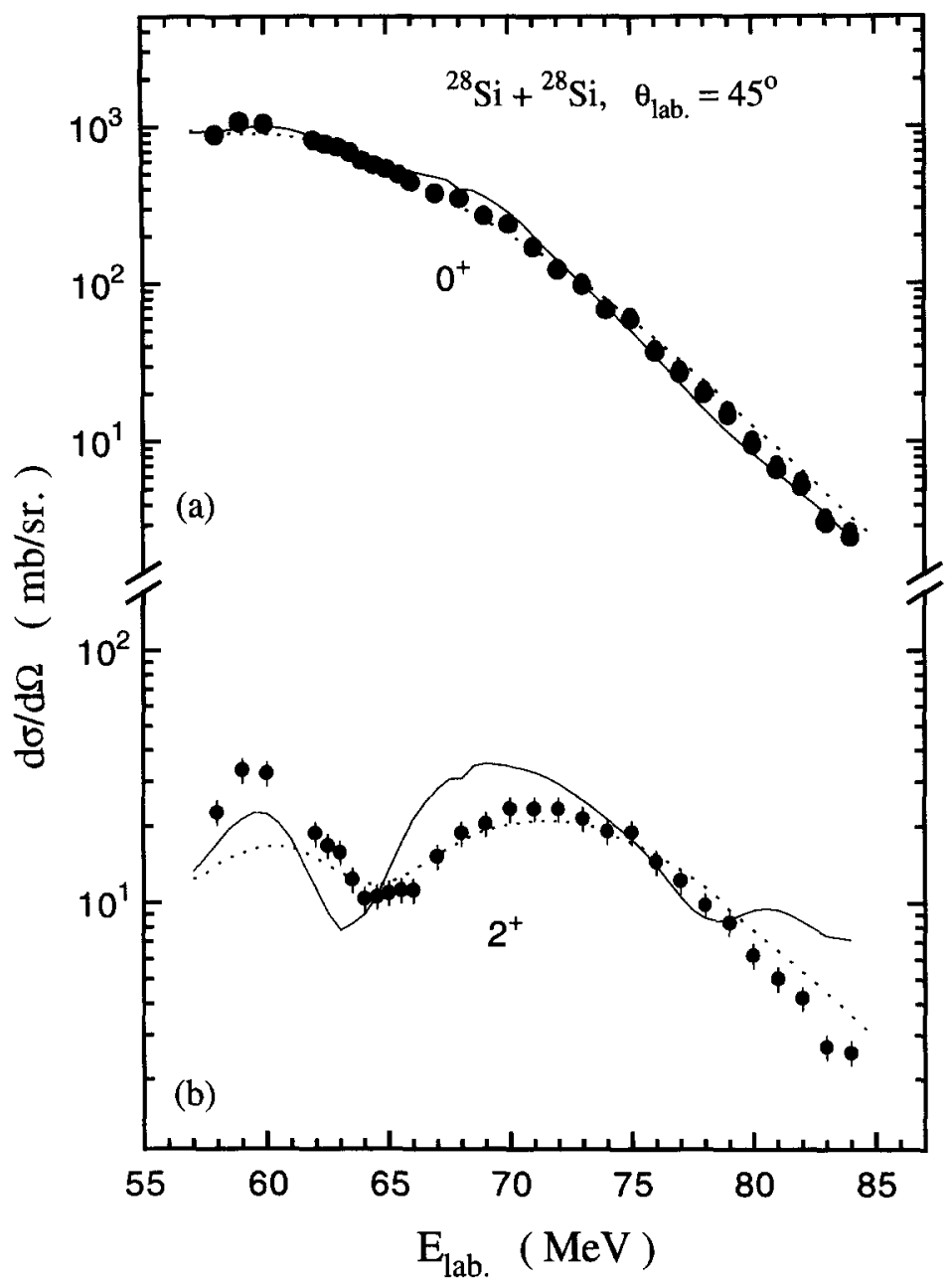

Fig. 8. Excitation function for elastic and inelastic $\left(2^{+}\right)$scattering of ${ }^{28} \mathrm{Si}$ on ${ }^{28} \mathrm{Si}$ system at $\theta_{\text {lah. }}=45^{\circ}$. Continuous curves are the results of coupled channels calculation including $0^{+}-2^{+}$coupling both target and projectile excitation and quadrupole reorientation. Solid curve represents the microscopic calculation and dotted curve is phenomenological (see text).

with contributions from channels $4^{+}$and $3^{-}$only. The calculated imaginary potential at and a few $\mathrm{MeV}$ above the Coulomb barrier becomes zero at some points in radial space. This reflects the very small diffusivity of the nuclear potentials near barrier. The energy and density-dependent real folded potential was calculated for different energy points. We observed a small energy dependence in the real part at the strong absorption radius also.

Fig. 8a shows the elastic excitation function at $\theta_{\text {c.m. }}=90^{\circ}$. For an identical particle system, the cross section is symmetric around this point. The solid curve in the figure represents the coupled channels calculation with $0^{+}-2^{+}$coupling and also $\left(2^{+}-2^{+}\right)$ reorientation coupling. The quality of the fit is reasonable.

We also performed the coupled channels calculation using the phenomenological po- 
tential described by parameter set of Table 1 . The calculated cross-sections at the higher energy (a few MeV above the Coulomb barrier) slightly overpredict the experimental cross sections, but generally the fit is satisfactory.

\subsection{Inelastic excitation function and Coulomb nuclear interference}

Inelastic cross sections were also obtained as the offshoot of the same calculation. Fig. 8b shows the prominent Coulomb nuclear dip in the experimental excitation function. The theoretical prediction with the microscopically calculated potential including $0^{+}-2^{+}$coupling and quadrupole reorientation coupling does not reproduce the experimental data very well. One possible reason for this discrepancy might be that the way we derive the coupling potential, the imaginary part may not be completely proper at and around the barrier energies. It was observed that at low energy absorption was vanishing at a few radial points. As the effect of the Coulomb excitation of other channels also comes through the coupling potential, so the absence of that absorption might be the reason for the low cross section in the theoretical calculation at the low energy.

Coupled channels calculations $\left(0^{+}-2^{+}\right.$coupling) with a phenomenological potential (Table 1) and reorientation coupling (oblate deformation) reproduce the cross section above the barrier energy within experimental error. At the low energy side the magnitude of the predicted cross-section is about a factor of two less than the experiment. This discrepancy has also been observed by Emiling et al. [24] in their inelastic scattering data at 66 and $68 \mathrm{MeV}$. Below and at the barrier, the inelastic cross section is mostly dominated by the Coulomb excitation. The effect of nuclear reorientation and absorption due to the nuclear process is negligible. The calculated value of the inelastic cross-section can be increased by introduction of a large Coulomb reorientation term. In Fig. 9a show the comparison between the calculations with prolate and oblate deformation.

To understand this we decompose the contribution of the Coulomb excitation from the nuclear coupling. Fig. $9 \mathrm{~b}$ shows the Coulomb and the nuclear contribution in the inelastic cross-section by dotted and dash-dot-dot curves respectively. It should be noted that the observed dip cannot possibly be explained by changing the magnitude of the nuclear amplitude, a change in phase might be needed. It is clear that just the inclusion of the $0^{+}-2^{+}$coupling along with the change in the real and imaginary potential as well as the nuclear strength parameter $\left(\beta_{02}\right)$ will not suffice to reproduce the observed dip. The changing of those parameters simply change the magnitude of the cross section leaving the shape of the excitation function unchanged. Therefore the only possible way to do this is by changing the reorientation coupling.

As an alternative scheme to fit the Coulomb nuclear interference region in the excitation function, the following procedure was adopted. The matrix element associated with the reorientation term $\left(\beta_{22}\right)$ is given an energy dependence of the form

$$
\left\langle 2^{+}\|\mathrm{E} 2\| 2^{+}\right\rangle=\left[\sqrt{2} A \sigma^{-3 / 2} \exp \left(-\frac{2\left(E-E_{0}\right)^{2}}{\sigma^{2}}\right)+1\right]\left\langle 2^{+}\|E 2\| 2^{+}\right\rangle_{0}
$$



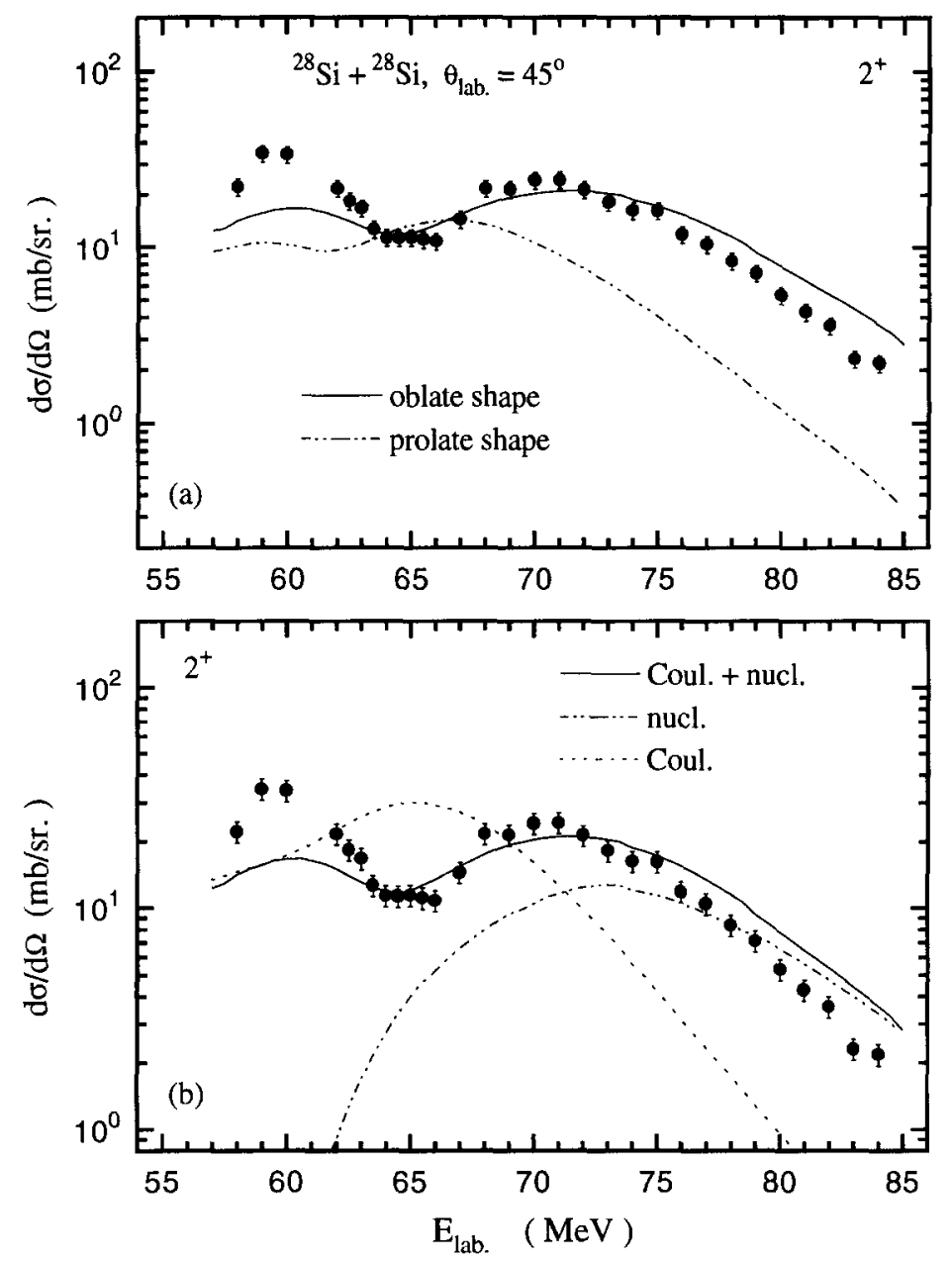

Fig. 9. Calculations with prolate and oblate shapes are shown in (a). The contribution of the Coulomb excitation and the nuclear coupling are shown in (b).

which is a Gaussian form, $\left\langle 2^{+}\|E 2\| 2^{+}\right\rangle_{0}$ being the energy independent term. The expression is similar to the energy dependent real potential $U(r, E)=V(r)+\Delta V(r, E)$, $\Delta V$ being the polarisation potential. The parameters were obtained through a fitting procedure and the values arrived at are: $\sigma$ : width of the distribution: $11.53 \mathrm{MeV}, A$ : area under the Gaussian: $71.37 \mathrm{MeV}^{3 / 2}, E_{0}$ : centroid: $55.61 \mathrm{MeV}$. The resulting fit is shown in the Fig. 10. The quality of the fit is fairly satisfactory except the low energy peak where it misses the data by about $30 \%$.

The physical significance of the energy dependence is as follows. The static quadrupole moment being a structure dependent quantity cannot possibly have any dependence on the projectile energy. However, the corresponding matrix element denoting off-diagonal channel coupling potential may have an energy dependence due to the same reason which causes the energy dependence in the diagonal optical potential. Indeed we have 


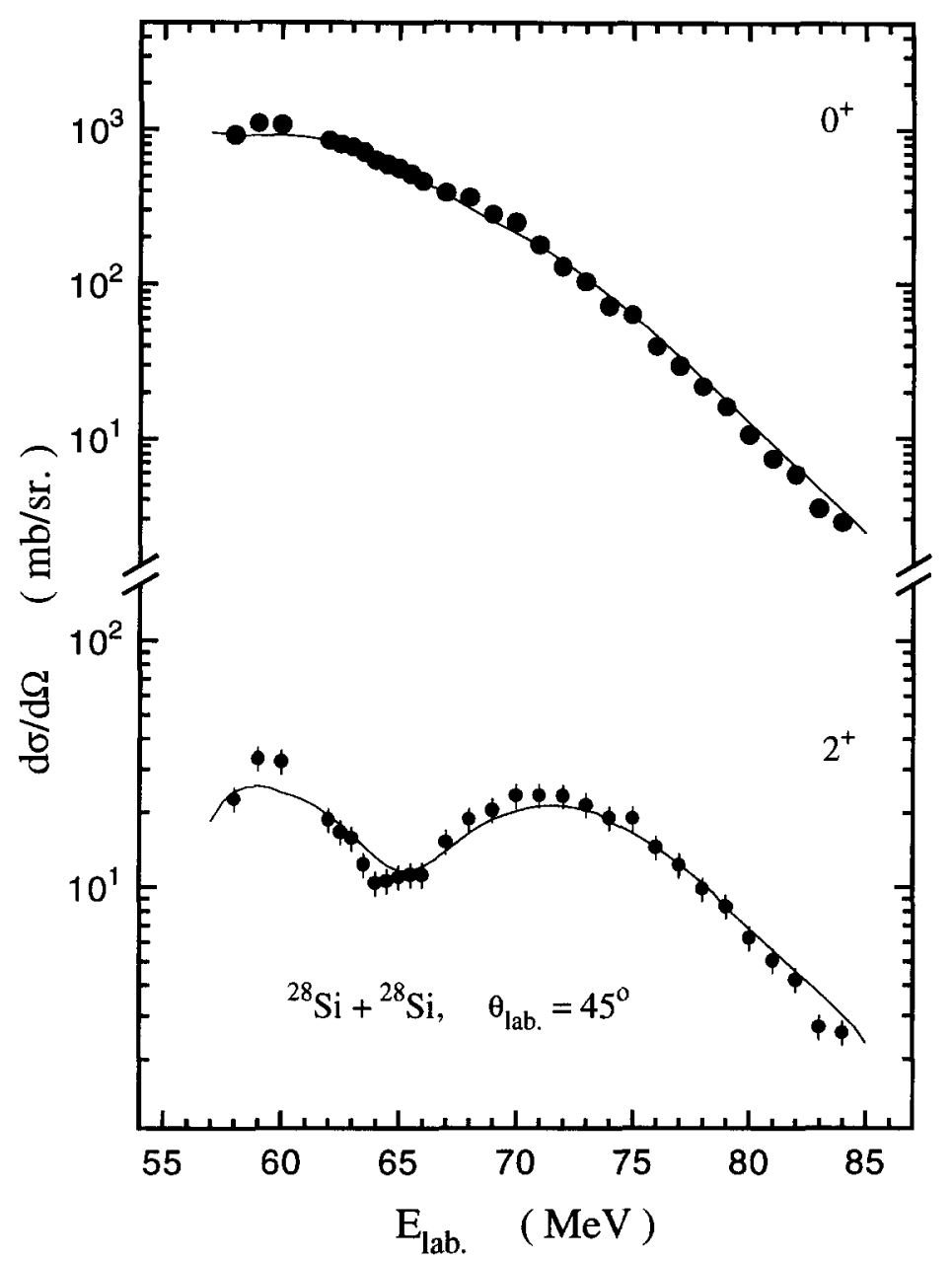

Fig. 10. Excitation functions fit with an energy dependent reorientation coupling (see text).

tried to keep a similar Gaussian form for this transition potential as in the real diagonal optical potential. Energy dependence in the reorientation coupling has been previously suggested [20] though a detailed fitting as in our work has never been attempted.

\section{Conclusions}

The elastic scattering angular distribution for ${ }^{28} \mathrm{Si}+{ }^{28} \mathrm{Si}$ were measured at 63 and $72 \mathrm{MeV}$ energies. The inelastic angular distribution was measured at $72 \mathrm{MeV}$. Around the Coulomb barrier from 58 to $84 \mathrm{MeV}$ the excitation functions for elastic and inelastic $\left(2^{+}\right)$were also measured. The data were analysed in a systematic way by using a phenomenological potential and microscopically derived potential from basic nucleusnucleus interaction. Calculated cross sections obtained from these approaches show 
very good agreement with the experimental angular distribution of elastic scattering with reasonable fits to inelastic scattering. But the Coulomb nuclear interference dip observed in the excitation function of inelastic $\left(2^{+}\right)$state is not described well. Observed enhanced cross section in the inelastic excitation function at and below the Coulomb barrier remained unexplained in our calculation. At the higher energy, $78 \mathrm{MeV}$ and above (a few $\mathrm{MeV}$ above the Coulomb barrier) the theoretically derived cross section from the microscopic potential shows a different pattern from the experimental cross section. It is likely that above the Coulomb barrier more number of channels are contributing to the absorption which we have not taken into account. A more elaborate calculation of the imaginary potential including explicit coupling of those channels is probably required. Addition of the energy dependent re-orientation coupling term improves the fit and lends support to the hypothesis that there is something significant missing in the channel coupling description as it is applied in this case.

Throughout our calculations we neglected the contribution of transfer coupling which may play a significant role just above the Coulomb barrier. The imaginary potential is derived by including only the inelastic scattering to $2^{+}, 3^{-}$and $4^{+}$states and the transfer reaction channels are not considered. It seems likely that inclusion of such channels might lead to the calculated imaginary potential to extend further out in the radial space. Transfer gets significant contributions from the tail of the wave function of the bound particle. The phenomenological potential which extends further out (set CCII) (thus perhaps mimicking same transfer) fits the data somewhat better. To include transfer channels in a proper calculation for microscopic absorptive potential, appropriate spectroscopic amplitudes have also to be used.

The major aspect which needs further refinement is the imaginary transition potential. We have simply taken the derivative of the imaginary diagonal potential obtained microscopically and used it as the transition potential. Perhaps an alternative procedure can be the use of a complex two-body effective interaction like JLM [9] and then obtain the imaginary transition potential by folding the imaginary part of the complex effective interaction with the transition density as in the case of the real transition potential. A second procedure could be to use appropriate shell-model (or RPA) wave function for the intermediate states $\left(2^{+}, 3^{-}, 4^{+}\right)$in the calculation of the imaginary potential along with appropriate $V_{\text {eff. }}$. The simplified collective form factor Eq. (22) might not be adequate.

Finally, we conclude that microscopic complex potentials can now describe the elastic data very well and predict general trends of the inelastic angular distribution. The more sensitive Coulomb nuclear interference is yet to be described satisfactorily by this procedure.

\section{Acknowledgements}

The authors are indebted to the NSC Pelletron group for their help and cooperation. We particularly thank Prof. C.V.K. Baba for many valuable discussions. We also thank 
N.M. Badiger for his help in part of the experiment.

\section{References}

[1] G.R. Satchler, Direct Nuclear Reactions (Oxford Univ. Press., Oxford, 1983).

[2] G.R. Satchler and W.G. Love, Phys. Rep. 55c (1979) 183.

[3] R.A. Broglia, G. Pollarolo and A. Winther, Nucl. Phys. A 36 (1981) 307.

[4] G. Pollarolo, R.A. Broglia and A. Winther, Nucl. Phys. A 406 (1983) 369.

[5] Fl. Stancu and D.M. Brink, Phys. Rev. C 25 (1982) 2450; C 32 (1985) 1937.

[6] D.M. Brink and Fl. Stancu, Phys. Rev. C 30 (1984) 1904.

[7] M. Trefz, A. Faessler and W.H. Dickhoff, Nucl. Phys. A 443 (1985) 499.

[8] M. Rashdan, A. Faessler, M. Ismall and W. Wadia, Nucl. Phys. A 466 (1987) 439;

M. Rashdan, A. Faessler, N. Ohtsuka, R. Linden, W. Wadia and M. Ismall, Z. Phys. A 330 (1988) 417.

[9] J.P. Jeukenne, A. Lejeune and C. Mahaux, Phys. Rev. C 16 (1977) 80.

[10] N. Vinh Mau, Nucl. Phys. A 457 (1986) 413, A 470 (1987) 406; Inst. Phys. conf. ser. 110 (1990) 1.

[11] N. Vinh Mau, J.C. Pacheco, J.L. Ferrero and R. Bilwes, Nucl. Phys. A 560 (1993) 879;

B. Bilwes, R. Bilwes, N. Vinh Mau, J.L. Ferrero and J.C. Pacheco, Nucl. Phys. A 526 (1991) 292.

[12] H. Feshbash, Ann. Phys. 5 (1958) 357; 19 (1962) 287.

[13] N. Vinh Mau, J.L. Ferrero, J.C. Pacheco and B. Bilwes, Nucl. Phys. A 531 (1991) 435.

[14] J.C. Pacheco, Bilwes, F. Sánchez, J.A. Ruiz, J. Díaz, J.L. Ferrero and D. Kadi-Hanifi, Nucl. Phys. A 588 (1995) 537.

[15] J.M. Barrigon, A. Baeza, J.L. Ferrero, J.C. Pacheco, B. Bilwes and R. Bilwes, Nucl. Phys. A 545 (1992) 720.

[16] V. Oberacker, H. Holm and W. Scheid, Phys. Rev. C 10 (1974) 1917.

[17] S. Landowne, A. Vitturi, Treatise on Heavy-Ion Science, Vol. 1 (Plenum, New York, 1984) p. 355.

[18] E.M. Takagui, G.R. Satchler, H. Takai, K. Koide and O. Dietzcsh, Nucl. Phys. A 514 (1990) 120.

[19] G.R. Satchler, Phys. Rep. 199 (1991) 147.

[20] M.J. Smithson, J.S. Lilley, M.A. Nagarajan, P.V. Drumm, R.A. Cunningham, B.R. Fulton and I.J. Thompson, Nucl. Phys. A 517 (1990) 193.

[21] P. Tikkanen, J. Keinonen and A. Kangasmäki, Phys. Rev. C 47 (1993) 145

[22] B.H. Windenthal, Bull. Am. Phys. Soc. 27 (1982) 725.

[23] A.J. Ferguson, O. Häusser, A.B. McDonald and T.K. Alexander, ANL Report no. ANL-7837, p. 187.

[24] H. Emling, R. Nowotny, D. Pelte, G. Schrieder and W. Weidenmeier, Nucl. Phys. A 239 (1975) 172.

[25| S. Mandal, T. Madhusoodhanan, A. Tripathi, D. Kabiraj, S. Ghosh, A. Mandal, D.K. Avasthi and S.K. Datta, Proc. DAE. Symp., Nucl. Phys. B 36 (1993) 438.

[26] J. Raynel, Computer program ECIS94, unpublished.

[27] J.J.A. Zalmstra, M.N. Harakeh and J.F.A. Van Hienen, Nucl. Phys. A 535 (1991) 23.

[28] R.H. Spear, Phys. Rep. 73 (1981) 369.

129] B.A. Brown, R. Radhi and B.H. Wildenthal, Phys. Rep. 101 (1983) 313.

[30] Dao T. Khoa and O.M. Knyazkov, Z. Phys. A 328 (1987) 67.

[31] Dao T. Khoa, W. von Oertzen and H.G. Bohlen, Phys. Rev. C 49 (1994) 1652.

[32] G. Bertsch, J. Borysowicz, H. McManus and W.G. Love, Nucl. Phys. A 284 (1977) 399.

[33] G.C. Li, M.R. Yeariam and I. Sick, Phys. Rev. C 9 (1974) 1861.

[34] P.D. Kunz, Computer code DWUCK4, unpublished.

135| P.J. Brussaard and P.W.M. Glaudemans, Shell Model Applications in Nuclear Physics (North Holland, Amsterdam, 1977).

[36] R.A. Broglia and A. Winther, Heavy ion reactions, Vol, 1 (Addison-Wesley, 1991), p. 116

[37] I.J. Thompson, Comp. Phys. Rep. 7 (1988) 167.

[38] G.R. Satchler, M.A. Nagarajan, J.S. Lilley and I.J. Thompson, Ann. Phys. 178 (1987) 110. 